\title{
Capacidad de asimilación de las competencias laborales en la industria de la comunicación gráfica ${ }^{1}$
}

\author{
LUZ ALEXANDRA MONTOYA \\ Ph.D. \\ Universidad Nacional de Colombia \\ lamontoyar@unal.edu.co \\ Profesora Asociada, Facultad de Ciencias \\ Económicas, Universidad Nacional de \\ Colombia, Investigadoragrupo BioGestion.
}

\author{
CARMEN ALICIA AMAYA \\ Universidad Nacional de Colombia \\ carmenaamaya@yahoo.com \\ Administradora de Empresas, Investigadora \\ BioGestión, proyecto Competencias Laborales \\ Cigraf,Universidad Nacional deColombia
}

\author{
OSCAR FERNANDO \\ CASTELLANOS Ph.D. \\ Universidad Nacional de Colombia \\ ofcastellanosd@unal.edu.co \\ Profesor Asociado, Facultad de Ingeniería, \\ Universidad Nacional de Colombia. \\ Coordinadorgrupo BioGestión
}

\begin{abstract}
Resumen ${ }^{2}$
El presente documento expone las principales características del desarrollo de un estudio exploratorio para la industria de comunicación gráfica, en el que se buscó analizar la posibilidad de asimilación de las empresas de un programa de formación, capacitación y evaluación de un sistema de competencias. En primer lugar se define la necesidad de un modelo de competencias para permitir que el sector sea más dinámico y sostenible como un modelo de gestión del talento humano y organizacional, después se exponen las competencias y se formula un modelo de investigación con una muestra de empresas del sector para así determinar cuáles son los requerimientos del mismo en el tema.
\end{abstract}

\section{Palabras Claves}

Estudio de mercados, competencias laborales, industria gráfica, percepción de los empresarios.

\section{Abstract}

This document describes the main features of the development of an exploratory study for the graphic arts industry where the possibility of assimilation of companies in a training program, training and evaluation of a competitive system, was analyzed. First, the study defines the need for a competency model to allows the sector to be more dynamic and sustainable as a management model of human and organizational talent, exposing the skills and formulating a research model with a sample of sector's companies in order to determine which are the requirements in the field.

\section{Key words}

Market research, job skills, graphic industry, perception of entrepreneurs.
Fecha de recepción: 1 de junio de 2010

Fecha de aprobación: 7 de julio de 2010

\section{Introducción}

La participación en un mercado internacional cada vez más competitivo e integrado, demanda de las organizaciones un cambio, en el cual los elementos como la incorporación continua de ciencia y tecnología en sus estructuras cobra una mayor importancia, así como el desarrollo de programas encaminados a la calidad en los productos y procesos; y muy especialmente, la gestión de un talento humano integral, es decir, trabajadores creativos, proactivos, permanentemente actualizados, con las actitudes y aptitudes, que no solo impriman valor agregado a productos y servicios, haciendo a las empresas en las que trabajan cada vez más competitivas, sino aportando de sí, el potencial necesario para hacer de cada empresa un sistema integral con un tejido social homogéneo y sostenible.

La industria de las artes gráficas, en el mundo (CPISC, 2008; Romano, 2003) pero particularmente en Colombia, no es ajena a esta dinámica, en especial, porque se ha considerado como un eslabón intermedio en una de las cadenas productivas con mayor importancia en el país, donde se encuentra en primer lugar la industria papelera, las tintas, los proveedores de máquinas, los equipos e insumos; en la parte intermedia: las empresas que imprimen en diferentes sistemas, y por último, los clientes, que son los editores de libros, revistas, las empresas de empaques, las agencias de publicidad y los sectores de manufactura

\footnotetext{
1. Investigación realizada en el marco del proyecto Generación de un sistema de información para Cigraf (Centro de Desarrollo Tecnológico para la Competitividad de la Industria de la Comunicación Gráfica), Análisis de competencias y desarrollo de programas curriculares basados en competencias laborales para la industria gráfica, desarrollado por la Coordinación Nacional de Extensión de la Universidad Nacional de Colombia, en la cual participaron los autores.

2. Agradecimientos: los autores del estudio manifiestan su agradecimiento al Cigraf, promotor del estudio y a las empresas participantes: Imprenta Nacional de Colombia, Legislación Económica SA, Printer Colombiana SA, Tecimpre SA, Gráficas Mundial Ltda., Sociedad Bíblica Colombiana, Prooffset editorial Ltda., Zetta Comunicadores Ltda., SI Servicios Impresos Ltda., Gráficas Colorama Ltda., CTP.COM, Gráficas Ducal Ltda., Ramírez Impresores Ltda.., Editorial Linotipia Bolívar, Ediciones Ántropos Ltda., Asesores Editoriales Ltda., Velto Ltda., Xpress Estudio Gráfico Digital, Litografía Sánchez M. Ltda., Exicartón SA, Editorial Gente Nueva, Editora Guadalupe Ltda.
} 
y construcción, entre otros. Esta industria tiene en su factor humano el principal y más importante pilar competitivo (Dharavath, 2004); de su desempeño, depende en gran medida el éxito o el fracaso, de la cadena en general (RedGráfica, 2007).

Ante el desafío que impone la realidad organizacional, en esta industria ha surgido una preocupación por el recurso humano y todo lo que le atañe. La mirada no solo es de quienes dirigen las empresas, sino de aquellas instituciones que sirven de apoyo a su funcionamiento. De esta manera, se enfoca en las problemáticas relacionadas con las personas, especialmente en el gran abismo que separa el mundo académico del laboral, hecho que ocasiona dificultades en este último, por causa de malos aprendizajes, ya sea en las aulas o en el contexto del trabajo y que se reflejan no solo en productos de mala calidad, sino que además repercute en el desarrollo individual y colectivo de las personas $\mathrm{y}$, por ende, en la dinámica productiva y social de las empresas.

El dominio del conocimiento por parte de los trabajadores y su precisa aplicación en cada actividad desempeñada, se ha convertido en una condición sin la cual no es posible para una empresa de la industria de la comunicación gráfica (Catalano et ál, 2010; Candiano, 2005) permanecer en la actualidad. Por ello, el establecimiento de Olos perfiles que se requieren en cada puesto de trabajo, así como de las competencias personales y laborales (Cardona, 2003), que debe tener la persona que lo ocupa o que aspire a ocuparlo, es una labor que se hace cada vez más importante. Y, más fundamental aún, se proponen los procesos de evaluación y formación, que han pasado casi inadvertidos en las políticas de gestión del factor humano, pues no se encuentran claramente sustentados sobre las competencias requeridas de una persona y las que realmente tiene, y que evidentemente, son procesos cruciales en la dinámica de las empresas de esta industria (Mertens, 2000 y 1996).

Es por ello que todos estos desarrollos, desde la gestión del recurso humano promueven sistemas en los que los empleados puedan trabajar mejor, una de las dinámicas que se ha validado es la implementación de un sistema de competencias, el cual busca ser además de un sistema de estandarización de las habilidades y el conocimiento, un sistema de calidad que busca que la empresa sea más sostenible en el mediano y largo plazos.

En Colombia, entidades como el SENA, han promovido el estudio de las competencias laborales y la estructuración de un marco normativo que les den un direccionamiento hacia la estandarización, como base para un proyecto que pretende articular toda la oferta de educación técnica en el país; de igual forma, algunas compañías han adoptado modelos similares para estructurar sus programas de capacitación internos. A pesar de estas iniciativas, el tema es aún poco explorado y aplicado en el contexto empresarial colombiano, especialmente en la industria de la comunicación gráfica, en la que no existen experiencias documentadas de programas de formación y evaluación que permitan articular un sistema general de competencias laborales para el sector (Cinterfor/ort, 2010 y 2001; Conocer, 1997; Norton, 2008).

Las competencias laborales hacen referencia a una forma de gestión del recurso humano basada en las habilidades que los trabajadores poseen, y que van más allá de los conocimientos que se presume tienen. Así, se dice que

posee competencia profesional quien dispone de los conocimientos, destrezas y aptitudes necesarios para ejercer una profesión (capacidades profesionales), puede resolver problemas profesionalles de forma autónoma y flexible (cualificación), y está capacitado para colaborar en su entorno profesional y en la organización del trabajo (Cariola, 1997, p. 63).

Además,

las competencias son una toma de responsabilididad personal del asalariado frente a las situaciones productivas, lo cual implica una actitud social más que un conjunto de conocimientos profesionales; y... un ejercicio sistemático de reflexibilidad en el trabajo, entendido como un distanciamiento crítico de sul trabajo, de la forma de hacerlo y de los conocimientos que moviliza (Novick, 1997: 20).

Bajo este concepto, se han diseñado modelos para elaborar programas de evaluación, formación y 
certificación en competencias laborales. La competencia ha sido definida como la característica que adquiere en forma sistemática una persona y que está directamente relacionada con una actuación eficiente en su puesto de trabajo, puede ser: conocimientos adquiridos, valores profundamente arraigados, habilidades prácticas o rasgos de comportamiento que definen la forma de reaccionar de las personas (Barrios, 2009; Miranda, 2006).

El Cigraf (Centro de Desarrollo Tecnológico para la Competitividad de la Industria de la Comunicación Gráfica) ha emprendido la elaboración de un modelo de formación y evaluación que se adapte a los requerimientos de la industria gráfica y que procure el mejoramiento de la calidad de la formación de los trabajadores vinculados a dichas empresas. En el avance de dicho proceso se hace necesario conocer la realidad concreta en la que se desarrolla el trabajo, las necesidades específicas de formación que poseen las empresas, así como la percepción y disposición que tienen a implementar un sistema de competencias laborales que involucra, necesariamente, los procesos de evaluación y formación, tema particular de análisis en la presente investigación.

\section{Metodología}

Para esta investigación, se seleccionaron un conjunto de 22 empresas de diferentes tamaños que manifestaron su disponibilidad a participar de la investigación, en cada una de ellas se aplicó un total de tres cuestionarios que corresponden cada uno, a un miembro de cada nivel (operario, supervisor, gerente) organizacional, cabe anotar que en algunas empresas, el número de encuestas es mayor o menor, debido a las condiciones particulares de cada firma.

Las empresas fueron seleccionadas de los subsectores más importantes: preimpresión, impresión y acabados. Se les aplicó un instrumento que permitiera evaluar el estado de las competencias laborales y las necesidades específicas de formación, y así analizar los factores culturales que influyen en la generación y desarrollo de dichas competencias.

De los datos obtenidos en la etapa de aplicación o trabajo de campo, se realizó un análisis que permitiera diagnosticar el estado de las competencias laborales requeridas por las empresas del sector gráfico, en sus diferentes niveles jerárquicos, buscando con ello encontrar las necesidades más significativas de formación que tienen las personas, así como determinar los factores de la cultura organizacional (Suárez y Castellano, 2006), que influyen decisivamente en la alineación de dichas competencias en la búsqueda de una mayor productividad y competitividad de las organizaciones, como sistemas abiertos y en constante cambio. Esto con el fin de brindar los elementos necesarios para emprender acciones de formación, expresadas en programas y currículos que se materialicen en un modelo en competencias aplicable a las empresas concretas objeto de estudio, así como en el sector gráfico en general.

El proceso metodológico seguido para el desarrollo de la presente investigación se representa en la FiguRA 1. Esta metodología y sus pasos fueron definidas desde el inicio de la investigación con el fin de seguir un proceso riguroso, que permitiera conocer, en primer lugar, la situación actual de las competencias laborales que se requieren para desempeñar las diferentes actividades que componen los cargos en las empresas del sector gráfico colombiano; de otro lado, conocer las necesidades de capacitación de la muestra de empresas, la disposición a la implementación de un sistema de competencias laborales; y, finalmente, los aspectos culturales que pueden influir decisivamente en dicho proceso de implementación.

Cada uno de estos pasos o etapas se desarrollaron de manera precisa, procurando que la investigación fuera sistemática, basada en fenómenos observables de la realidad de las empresas seleccionadas; y crítica, es decir, buscando tratar de manera objetiva los resultados obtenidos. Finalmente, con esta metodología se pretendió que la presente investigación fuera válida, confiable y que cumpla ante todo con los objetivos para los cuales se realizó.

Como puede apreciarse la primera parte, etapa 1, se trata del avance preliminar de la investigación, allí se define la situación problemática, se hace la revisión bibliográfica, y a partir de ello y de las necesidades del sector se plantean las variables a evaluar, las cuales son específicamente de dos tipos: culturales y de capacitación. En esta etapa preliminar se realizaron los primeros modelos de cuestionario, diseñando 
Figura 1. Metodología general de la investigación

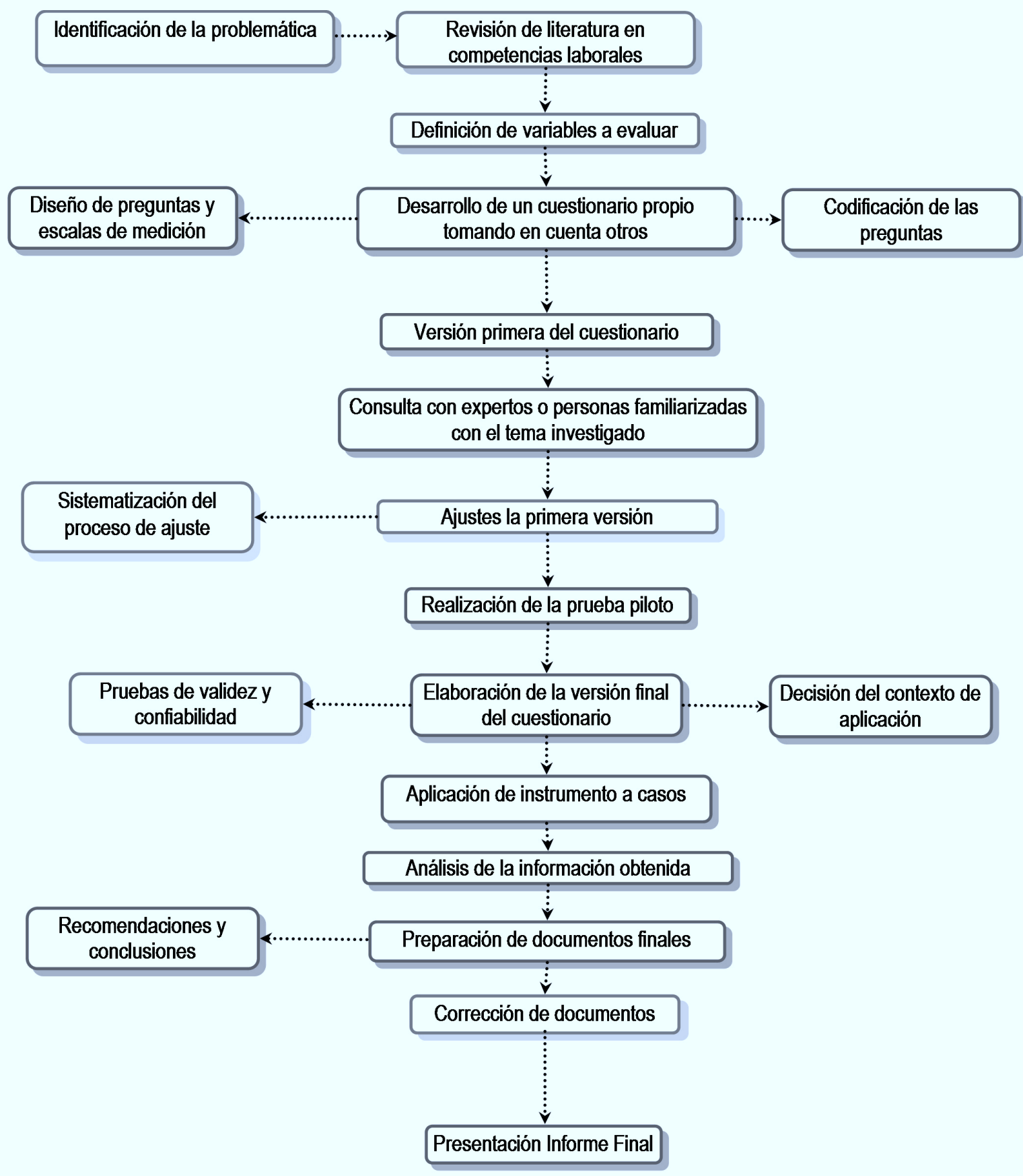

Preliminares

Diseño e implementación de una metodología de diagnóstico en competencias laborales

Evaluación

Análisis y presentación de información 
las preguntas, escalas de medición y su codificación. Posteriormente se siguió a la etapa 2, la cual se encargó de la evaluación del instrumento. En la etapa 3 se realizó el diseño e implementación de una metodología de diagnóstico en competencias laborales, en esta etapa se ejecuta la prueba piloto, la cual permite la sistematización y proceso de ajuste, se aplican las pruebas de validez y confiabilidad y se decide la elaboración de la versión final. Finalmente, en la etapa 4 , se producen los informes, a partir del análisis de la información obtenida (ver Figura 1).

\subsection{Selección de variables a evaluar}

El conjunto total de variables seleccionadas a fin de conocer la situación actual de la muestra de empresas del sector gráfico en Bogotá DC, en las competencias laborales requeridas para sus diferentes procesos productivos, así como hacer un acercamiento a los aspectos formales y culturales que establecen la disposición de las empresas para implementar un sistema de competencias laborales, fueron definidas teniendo en cuenta la mayor cantidad de las mismas que afectan el proceso, en especial se dividieron en dos grandes grupos: las primeras evaluaron la posibilidad de formación y aceptación de las competencias laborales, y un segundo grupo, de variables definidas como culturales estaban encargadas de conocer las dificultades que podría tener este proceso.

A continuación se describen las variables que se definieron para abordar el presente estudio (Tabla 1):

Tabla 1. Variables definidas para el estudio

\begin{tabular}{|c|l|}
\hline Variable & \multicolumn{1}{c|}{ Descripción } \\
\hline & $\begin{array}{l}\text { Las preguntas que constituyen esta } \\
\text { variable agrupan información básica } \\
\text { de las empresas que identifica a cada } \\
\text { una de ellas y a las personas que } \\
\text { respondieron la encuesta. Entre estos } \\
\text { aspectos se destaca el nivel de la } \\
\text { empresa al que pertenece la persona } \\
\text { que diligencia, variable que se } \\
\text { utilizará como criterio para clasificar } \\
\text { genanalizar la información obtenida. }\end{array}$ \\
\hline
\end{tabular}

\begin{tabular}{|c|c|}
\hline Variable & Descripción \\
\hline $\begin{array}{l}\text { Tamaño de } \\
\text { empresa }\end{array}$ & $\begin{array}{l}\text { Para clasificar el grupo de empresas } \\
\text { participantes en la investigación } \\
\text { se tuvo en cuenta la Ley } 590 \text { de } \\
\text { julio de 2000, que establece como } \\
\text { parámetros de referencia en el el } \\
\text { establecimiento del tamaño de las } \\
\text { empresas el número de empleados. }\end{array}$ \\
\hline $\begin{array}{l}\text { Productos y } \\
\text { servicios }\end{array}$ & $\begin{array}{l}\text { Esta variable pretende conocer los } \\
\text { productos y servicios que se ofrecen } \\
\text { y así determinar la frecuencia de } \\
\text { empresas que se encuentran para } \\
\text { cada uno. }\end{array}$ \\
\hline Calidad & $\begin{array}{l}\text { Se pretende conocer la relación entre } \\
\text { la experiencia de las empresas en un } \\
\text { sistema de calidad y la disponibilidad } \\
\text { a implementar un sistema de } \\
\text { competencias laborales. Se parte de } \\
\text { la base de que aquellas empresas } \\
\text { que ya han tenido experiencia en } \\
\text { la adquisición de certificados de } \\
\text { calidad tendrán mayor disponibilidad } \\
\text { a implementar un sistema de } \\
\text { competencias laborales. }\end{array}$ \\
\hline $\begin{array}{l}\text { Entorno } \\
\text { formal }\end{array}$ & $\begin{array}{l}\text { Esta variable busca medir, por un } \\
\text { lado, el estado de algunos elementos } \\
\text { formales del entorno interno en } \\
\text { las empresas que se relacionan } \\
\text { directamente con la gestión del factor } \\
\text { humano, como son la comunicación } \\
\text { y la participación en la toma de } \\
\text { decisiones; y por otro, se indaga en la } \\
\text { percepción que tienen los diferentes } \\
\text { miembros de las empresas de las } \\
\text { relaciones que se establecen con } \\
\text { agentes tanto internos como externos } \\
\text { a ella tales como clientes, proveedores, } \\
\text { distribuidores y empleados. }\end{array}$ \\
\hline $\begin{array}{c}\text { Elementos de } \\
\text { competencia } \\
\text { laboral }\end{array}$ & $\begin{array}{l}\text { Con este conjunto de preguntas } \\
\text { se busca conocer la percepción de } \\
\text { las personas encuestadas frente al } \\
\text { desempeño de los trabajadores en las } \\
\text { diferentes actividades que conforman } \\
\text { los procesos productivos. }\end{array}$ \\
\hline
\end{tabular}




\begin{tabular}{|c|c|}
\hline Variable & Descripción \\
\hline $\begin{array}{l}\text { Políticas de } \\
\text { capacitación }\end{array}$ & $\begin{array}{l}\text { Estas políticas se enfocan } \\
\text { específicamente en las características } \\
\text { de la capacitación de los niveles en } \\
\text { cada empresa, en su pertinencia, } \\
\text { frecuencia, lugar donde se recibe, } \\
\text { temas específicos, personas que la } \\
\text { imparten, dinero invertido, entre } \\
\text { otras; brindan la información } \\
\text { necesaria para determinar la } \\
\text { disposición que pueden llegar a tener } \\
\text { las empresas, para iniciar procesos } \\
\text { encaminados a la formación de su } \\
\text { personal en la implementación de un } \\
\text { sistema de competencias laborales. }\end{array}$ \\
\hline $\begin{array}{l}\text { Condiciones } \\
\text { de trabajo }\end{array}$ & $\begin{array}{l}\text { Son los elementos que caracterizan } \\
\text { la organización como espacio local, } \\
\text { donde se comparte un lugar físico } \\
\text { común en el cual se desarrollan } \\
\text { las actividades directamente } \\
\text { relacionadas con el trabajo. Estos } \\
\text { elementos evaluados dan razón de } \\
\text { las condiciones particulares que } \\
\text { rodean actualmente el desarrollo } \\
\text { de las competencias laborales en la } \\
\text { muestra de empresas, elementos a } \\
\text { tener en cuenta en el momento de } \\
\text { construir un modelo de formación } \\
\text { por competencias específico para el } \\
\text { sector de artes gráficas. }\end{array}$ \\
\hline $\begin{array}{l}\text { Evaluación de } \\
\text { desempeño }\end{array}$ & $\begin{array}{l}\text { La evaluación del rendimiento } \\
\text { puede ser una acción o conjunto de } \\
\text { acciones mediante las cuales, se logra } \\
\text { reconocer la contribución que hacen } \\
\text { las personas y su acción colectiva } \\
\text { a la organización. La información } \\
\text { obtenida permitirá conocer y analizar } \\
\text { la forma como se está desarrollando } \\
\text { la evaluación del trabajo en las } \\
\text { empresas; además, busca conocer } \\
\text { la percepción que tienen las } \\
\text { personas de los diferentes niveles } \\
\text { organizacionales, de los criterios de } \\
\text { dicho proceso y su disposición frente } \\
\text { al mismo. }\end{array}$ \\
\hline & \\
\hline
\end{tabular}

\begin{tabular}{|c|c|}
\hline Variable & Descripción \\
\hline $\begin{array}{c}\text { Cultura } \\
\text { organizacional }\end{array}$ & $\begin{array}{l}\text { Se define como: las transformaciones } \\
\text { que generan comportamientos } \\
\text { organizacionales que consolidan o } \\
\text { modifican valores institucionales; } \\
\text { crean costumbres, prácticas, } \\
\text { procedimientos, normas y conductas } \\
\text { entre los miembros de la corporación. } \\
\text { Por la importancia que tiene la } \\
\text { cultura para gestión humana en las } \\
\text { organizaciones, se incluye como } \\
\text { variable en el análisis de la presente } \\
\text { investigación, con el fin de determinar } \\
\text { los rasos particulares que muestra } \\
\text { en cada empresa y que facilitan o } \\
\text { dificultan la implementación de un } \\
\text { sistema de evaluación, formación } \\
\text { labrtificación en competencias } \\
\text { laborales. }\end{array}$ \\
\hline $\begin{array}{l}\text { Percepción } \\
\text { y actitud } \\
\text { frente a un } \\
\text { sistema de } \\
\text { competencias } \\
\text { laborales }\end{array}$ & $\begin{array}{l}\text { En esta variable se contemplan } \\
\text { aspectos como: experiencia de las } \\
\text { empresas en la implementación de un } \\
\text { sistema de esta clase, importancia de } \\
\text { iniciar la ejecución de dicho sistema, } \\
\text { importancia de la certificación de } \\
\text { los trabajadores, calificación de las } \\
\text { ventajas y desventajas que conlleva el } \\
\text { sistema para la empresa, disposición } \\
\text { a invertir y condiciones para la } \\
\text { implementación. }\end{array}$ \\
\hline $\begin{array}{c}\text { Percepción } \\
\text { instituciones } \\
\text { evaluadoras y } \\
\text { formadoras en } \\
\text { competencias } \\
\text { laborales } \\
\text { (Cigraf - SENA) }\end{array}$ & $\begin{array}{l}\text { El grupo de preguntas evaluadas } \\
\text { están enfocadas en conocer la } \\
\text { percepción que tienen las empresas } \\
\text { del Cigraf, no solo como centro de } \\
\text { desarrollo tecnológico, sino como } \\
\text { institución evaluadora y formadora } \\
\text { en competencias laborales en la } \\
\text { industria de la comunicación gráfica. }\end{array}$ \\
\hline
\end{tabular}

\subsection{Selección de la muestra}

La presente investigación toma como objeto de análisis una muestra de 22 empresas distribuidas en los tres tamaños: pequeñas, medianas y grandes (clasificación hecha teniendo como referencia el parámetro "número de empleados" de la Ley 590 de julio de 2000). Dieciséis empleados tuvieron la más pequeña y 439 
la más grande. Fueron seleccionadas por el Cigraf de un universo de setenta empresas aproximadamente, (la muestra fue de 30\%) en Bogotá. Las empresas elegidas mostraron disposición para participar en el estudio debido al interés que despertó en sus directivos el tema de las competencias laborales para la industria de la comunicación gráfica.

De los datos obtenidos en la etapa de aplicación o trabajo de campo, se realizó un análisis que permitió diagnosticar el estado de las competencias laborales requeridas por las empresas del sector gráfico, en sus diferentes niveles jerárquicos, buscando con ello encontrar las necesidades más significativas de formación que tienen las personas, así como determinar los factores de la cultura organizacional que influyen decisivamente en la alineación de dichas competencias en la búsqueda de una mayor productividad y competitividad de las organizaciones, como sistemas abierto y en constante cambio; todo esto con el fin de brindar al Cigraf los elementos necesarios para emprender acciones de formación, expresadas en programas y currículos que se materialicen en un modelo en competencias aplicable a las empresas concretas objeto de estudio, así como en el sector gráfico en general.

Con la información recogida se pudo concluir que las empresas de la muestra pertenecientes a la industria de la comunicación gráfica, cuentan con una población de trabajadores, en su mayoría operarios permanentes y una proporción considerable de administrativos, siendo el número de supervisores menor que los dos anteriores. Esta información lleva a considerar que al iniciar un proceso de implementación de un sistema de competencias laborales, requiriendo una demanda de capacitación permanente en este campo. En cuanto a la población de trabajadores temporales son empleados para procesos que no requieren mayor especialización, como la encuadernación y los acabados, constituyen un mercado potencial en el que se pueden buscar mecanismos para implementar el sistema, con el fin de brindar a estos trabajadores el proceso de certificación.

La Tabla 2 muestra la distribución de productos y servicios que tienen las empresas participantes en el estudio, se observa que la mayoría de empresas de la muestra se clasifican en las categorías libros y publicaciones, publicomerciales, revistas y periódicos, diseño y otros impresos editoriales, las demás categorías reportan frecuencias menores, e incluso nulas como es el caso de los juegos, categoría en la cual no se clasificó ninguna empresa.

Tabla 2. Productos y servicios

\begin{tabular}{|l|l|}
\hline \multicolumn{2}{|l|}{ Productos y servicios } \\
$\begin{array}{l}\text { - Artículos escolares y de } \\
\text { oficina }\end{array}$ & $\begin{array}{l}\text { - Productos publicitarios y } \\
\text { comerciales }\end{array}$ \\
- Empaques flexibles & - Revistas y periódicos \\
- Empaques rígidos & - Diseño \\
- Etiquetas & - Soporte \\
- Formas y valores & - Cartonería \\
- Juegos de salón & - Planchas para impresión \\
- Libros y publicaciones & - Preprensa digital \\
- Otros impresos editoriales & - Otro servicio \\
\hline
\end{tabular}

\section{Resultados y análisis}

Después de la aplicación del instrumento, se obtuvieron los puntajes de cada uno de los niveles existentes en las empresas, datos que constituyen en conjunto, el material necesario para la realización del análisis y la formulación de las conclusiones y recomendaciones posteriores.

La presentación de los resultados y su consiguiente análisis se hacen teniendo en cuenta el orden de las variables descritas. Fueron analizados tres niveles (operativo, técnico y profesional). Otro tipo de análisis se enfoca en el tamaño de las empresas, que profundiza de manera global en el conjunto de estas, por su tamaño, y permite conocer las tendencias en cada uno de los grupos (grande, mediana, pequeña) así como sus necesidades y percepción de un sistema de competencias laborales. 
Durante la investigación se observó en la muestra de empresas una tendencia a la producción editorial, los productos publicitarios y comerciales, así como las revistas y periódicos y una menor frecuencia de otro tipo de productos. Debido a esta diversidad de productos en estas empresas del sector, en la implementación de un sistema de competencias laborales es necesario tener en cuenta los productos o servicios que tiene cada una de dichas organizaciones para el diseño de los programas específicos de evaluación y formación, y obtener de esta forma, un producto más especializado y adaptado a las particularidades de cada empresa.

\subsection{Percepción y disposición a la implementación de un sistema de competencias laborales (SCL)}

Un sistema de competencias laborales (ScL) implica el conjunto de elementos, acciones y procedimientos encaminados a establecer mecanismos de definición, evaluación y certificación de competencia laboral, de conformidad con las normas establecidas, así como mecanismos de acreditación, con el propósito de otorgar reconocimiento formal de la competencia adquirida por parte de los individuos en el transcurso de su carrera productiva, independientemente del modo en que esta haya sido adquirida. Este proceso incluye también la certificación de calidad de la capacitación basada en competencias laborales (Ministerio de Educación de Chile, 2003: 8).

La implementación de un sistema de competencias laborales es un proceso de cambio en todas las áreas de las empresas, y requiere diferentes aspectos a tener en cuenta, en especial, la experiencia que en el tema han tenido las organizaciones, la disposición a su implementación, la percepción que tienen las personas de dicho sistema, así como la disponibilidad específica para iniciar este proceso. A continuación se presentan los resultados correspondientes a estos aspectos, que fueron evaluados en cada una de las empresas participantes en el estudio.

\section{a. Experiencia en la implementación de un SCL}

Solo el 18\% de las empresas han iniciado algún análisis de competencias en puestos de trabajo con el fin de implementar un sistema de competencias laborales. De cuatro empresas, únicamente dos, han estado asesoradas por alguna institución en dicho proceso de implementación.
Cabe destacar que las empresas que han tenido experiencia en la implementación de un sistema de competencias laborales son de diferentes tamaños, dejando ver que dicho proceso es susceptible de ser realizado en cualquier empresa. La información recopilada con estas preguntas, permite establecer el potencial que representa el conjunto de empresas restantes (82\%), que no han iniciado un proceso similar y que pueden estar muy interesadas en esta nueva posibilidad de cambio, a fin de mejorar tanto su productividad como su competitividad.

\section{b. Disposición a la implementación de un scL}

La implementación de un sistema de competencias laborales es un proceso en el que se debe contar con la percepción que tienen las personas de los diferentes niveles frente a dicho sistema, específicamente enfocado en la importancia de su implementación, así como la tiene el proceso de certificación. Cada nivel organizacional manifestó su percepción del sistema como se describe a continuación en los tres niveles.

\section{?] Nivel operativo}

Los resultados para los operarios en el conjunto de preguntas se presentan en la Figura 2, en la que se muestra por tamaño de empresa, los promedios y las desviaciones estándar para cada ítem. Se observa que los puntajes promedio totales para los tres grupos de empresas son iguales o superiores a 4.0, en todas las preguntas, información que refleja una percepción positiva de los operarios frente a la implementación de un sistema de competencias laborales.

Se aprecia también, que los operarios de las empresas grandes son quienes tienen una percepción más favorable, todos los puntajes alcanzan el tope máximo (5.0), ya que consideran importante la implementación de un SCL en sus empresas y estarían de acuerdo en que sea iniciado dicho proceso. En cuanto a la certificación, los operarios de este grupo de empresas lo consideran como un aspecto muy importante y estarían dispuestos a invertir en su implementación.

De otro lado, se observan desviaciones altas, en las empresas medianas especialmente, que indican alta variabilidad entre empresas del mismo tamaño, estos datos reflejan la particularidad que tiene cada una, así 
como la diferencia notoria que tiene la implementación de un sistema de competencias laborales en cada una de ellas.

Los operarios de los otros dos grupos de empresas (mediana y pequeña) se muestran en promedio un poco menos optimistas, aunque obtienen puntajes igualmente altos. Se destacan los puntajes de las empresas medianas que son los más bajos del total de empresas, casos en los que los operarios no se muestran decididos en la implementación de un ScL en sus empresas, así como también se muestran indecisos en el momento de invertir en el proceso.

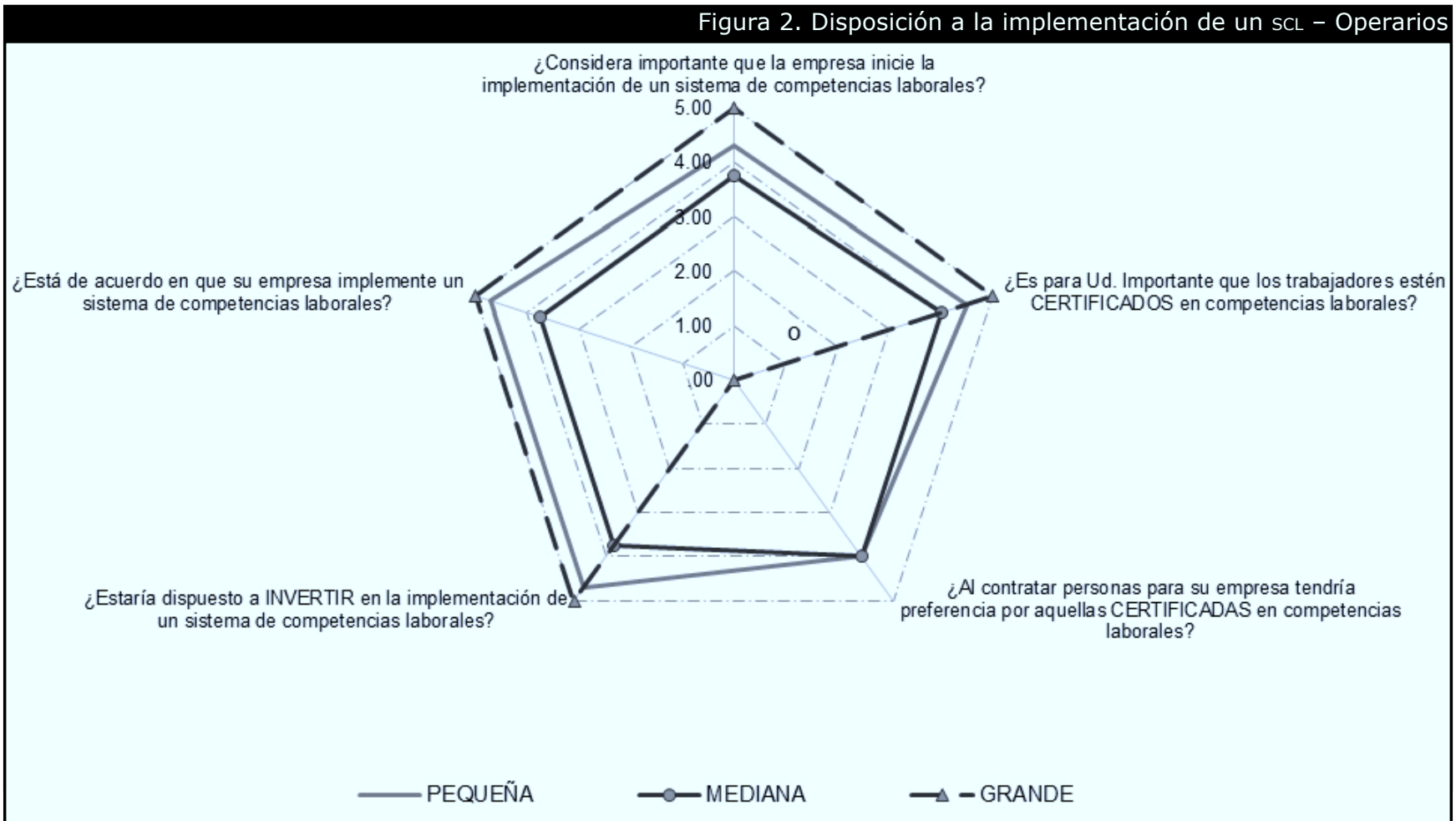

A pesar de lo anterior, en general, los operarios del total de empresas participantes en el estudio tienen expectativas favorables del sistema, además de considerarlo un proceso necesario, con el que verán mejorado su desempeño individual y de grupo.

\section{- Nivel supervisor}

La Figura 3 presenta con mayor claridad esta tendencia, los promedios totales en cada pregunta para los tres grupos de empresas (grande, mediana y pequeña) son altos y reflejan el interés que tienen los supervisores en el SCL. En las empresas medianas, las personas de este nivel tienen una percepción más favorable que los operarios de este mismo conjunto de organizaciones.

En todas las empresas, se considera importante la implementación del sistema y estarían dispuestos a invertir en él. Se destaca el eje que representa el valor de la certificación, aspecto en el que los supervisores se distancian un poco, y que refleja variabilidad en las percepciones de las personas, ya que no tiene la misma ponderación para todas.

En general para el total de empresas, las desviaciones son menores que en el nivel operativo, aunque siguen siendo altas, hecho que confirma la existencia de aspectos propios en cada empresa y nivel, que se deben tener en cuenta en el momento de implementar un SCL.

\section{- Nivel gerencial}

Se destacan los puntajes de las empresas grandes, que alcanzan valores máximos de la escala (1-5) y tienen desviaciones nulas, esto muestra la homogeneidad en las percepciones de los gerentes de estas empresas, en contraste con las empresas pequeñas, en las que a pesar de obtener puntajes altos, se observan desviaciones igualmente altas, indicador de variabilidad entre las empresas de este grupo. 
¿Considera importante que la empresa inicie la

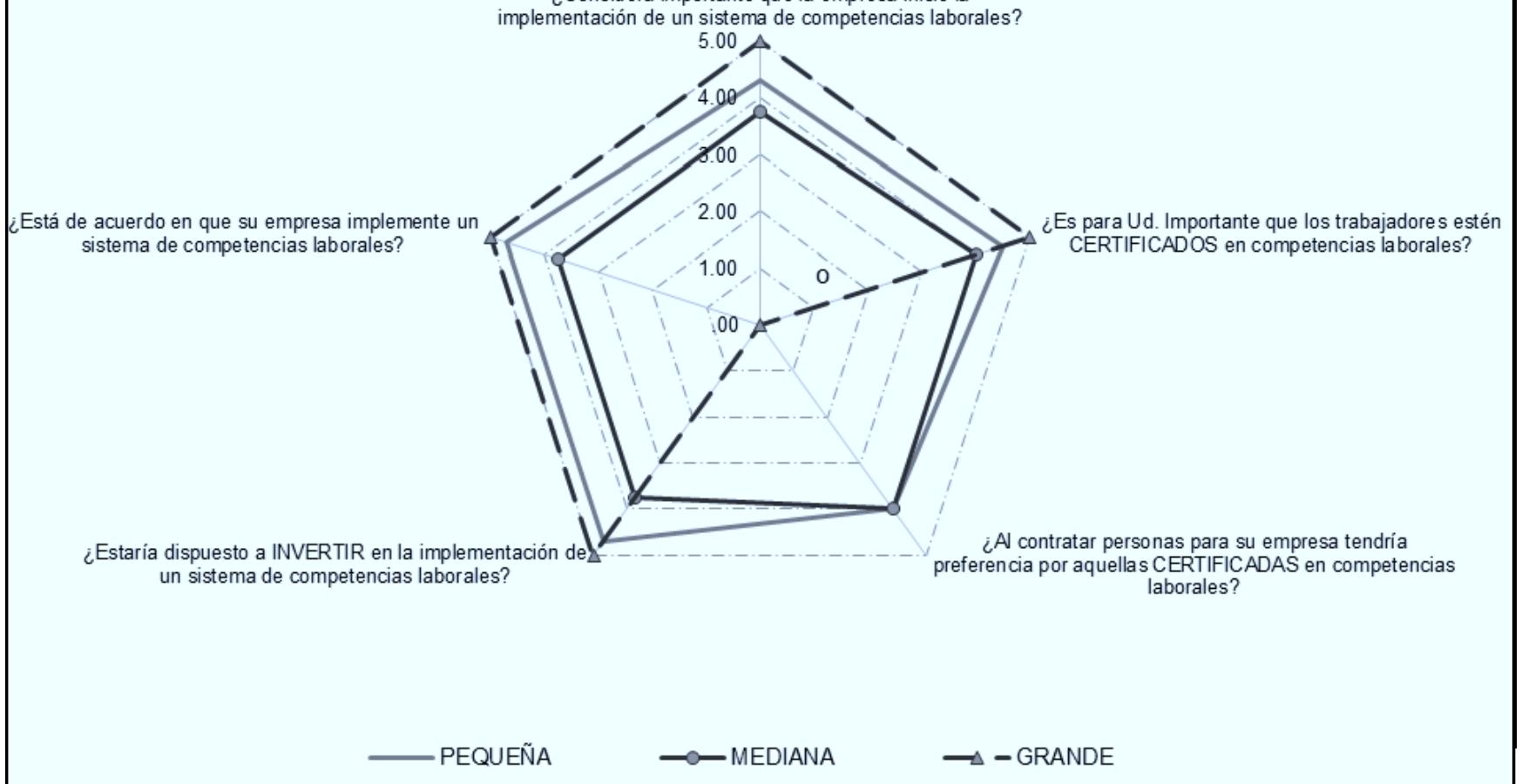

Con respecto a los otros dos niveles, el gerencial se muestra más interesado en invertir en un scL en las empresas medianas y grandes (puntajes promedio muy cercanos a 5.0). Por el contrario, los gerentes de las empresas pequeñas, reconocen la importancia que tiene el sistema, pero en cuanto a la inversión en el mismo, asignan puntajes que tienen alta variabilidad entre empresas y obtienen un puntaje promedio no muy alto (3.5).

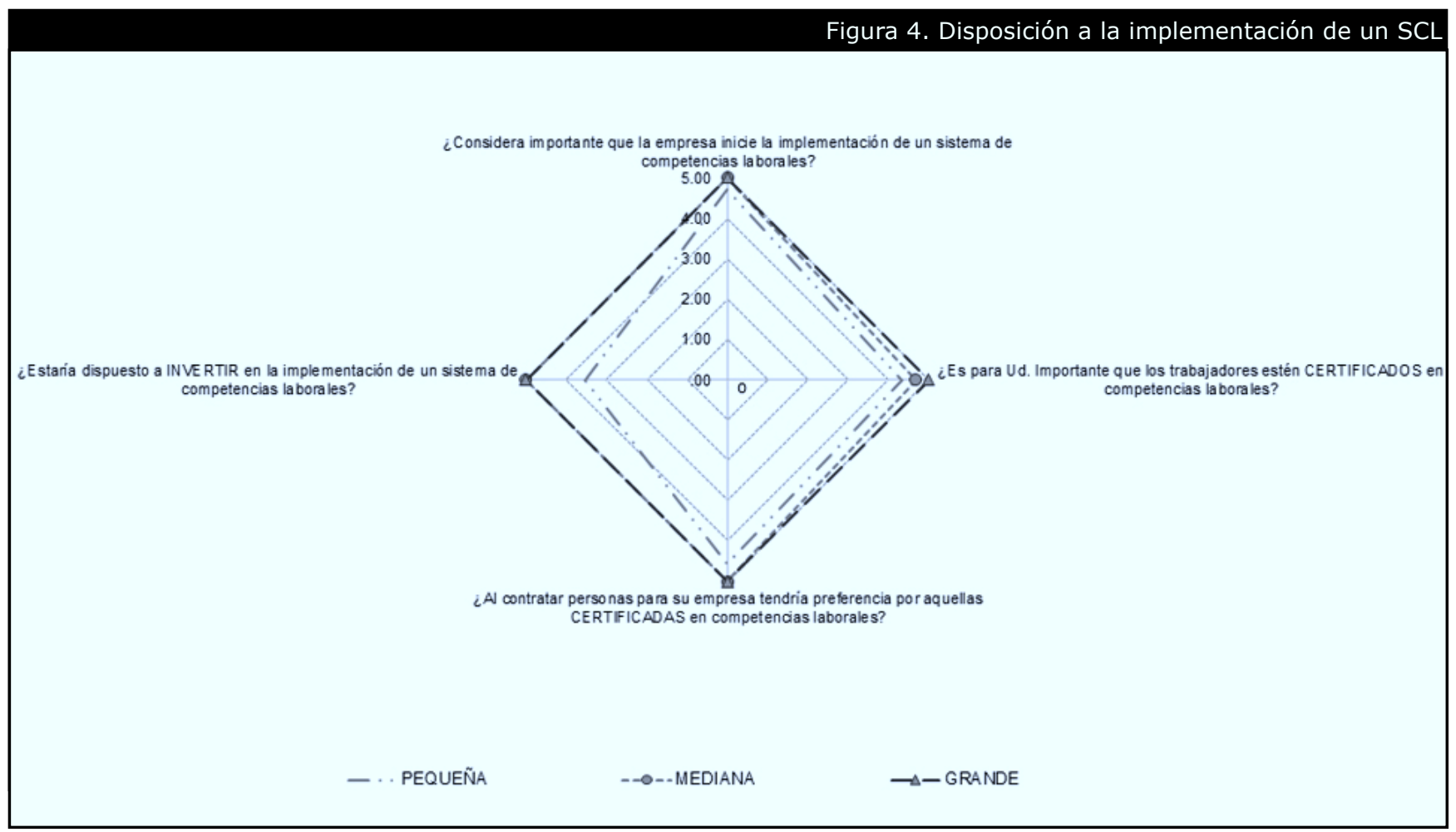




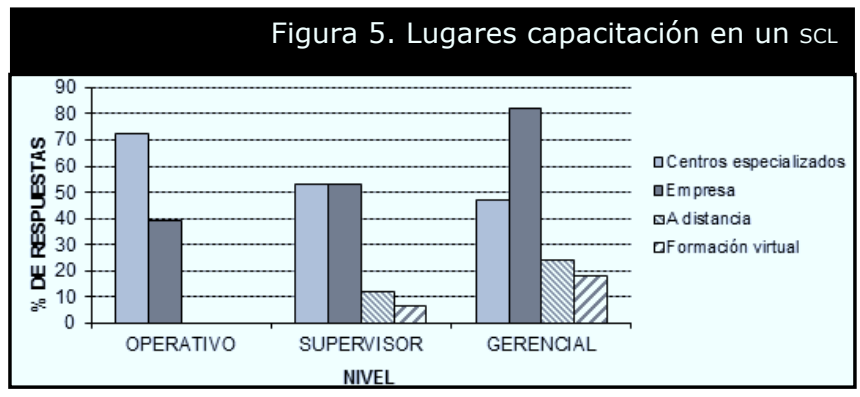

En la Figura 4 se ilustra lo ya descrito, y se observan los gerentes de las empresas medianas y grandes muy cercanas entre sí, asignando valores altos y poco variables a cada una de las preguntas; y en las pequeñas, las personas de este nivel reconocen la importancia del sistema y su implementación, pero se ven alejadas del resto de empresas en la inversión concreta en el sistema. Se refleja la conciencia que tienen algunos empresarios de que sus recursos son limitados y que no estarían en capacidad de implementar un sistema de dicha magnitud en sus organizaciones.

\section{c. Aspectos específicos de la implementación de un scL}

En este apartado del instrumento, se buscó obtener información específica sobre la disponibilidad de las empresas para implementar un ScL. Entre los aspectos tenidos en cuenta están: número de trabajadores a vincular en el proceso, lugares donde se debe recibir la formación, meses, días y horarios disponibles para esta implementación, la Figura 5 señala los posibles lugares en donde sería interesante realizar un proceso de formación en un scl.- Gerencial.

En general puede observarse en la Figura 5, que los operarios prefieren ir a centros especializados para su formación, en tanto que los gerentes optan más bien que se haga en la empresa. La formación virtual no es una alternativa para los operarios, pero sí está considerada por parte de los supervisores y los gerentes. Al realizar un promedio se desataca que la formación debería hacerse en la empresa y como segunda opción en los centros de formación.

Con respecto a los criterios de evaluación como medida de desempeño puede valorarse en la actitud del operativo en las empresas pequeñas y medianas, en tanto que en la empresa grande tiene mayor valor el desempeño y la actitud del supervisor. Solo las empresas grandes pueden valorar la actitud del operativo, en igual dimensión que el desempeño productivo gerencial y la actitud gerencial.

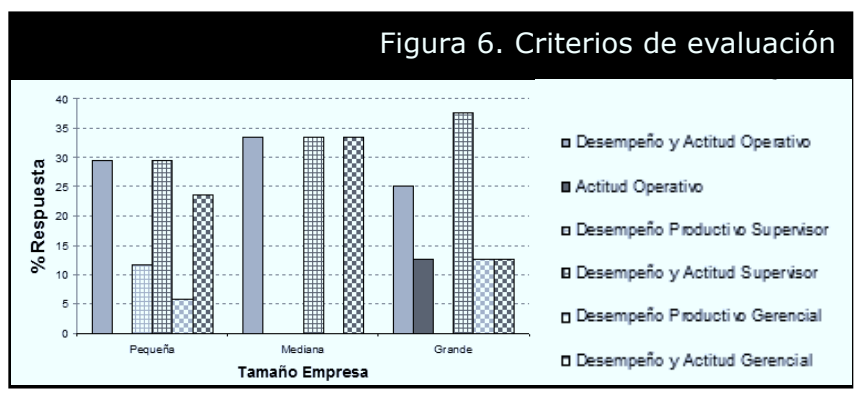

De igual forma se indagó sobre la evaluación como un indicador de competencia, de esta manera podría encontrarse el valor de un sistema de desarrollo de competencias, con los resultados de la Figura 7.

El análisis señala el alto puntaje que tanto el mando operativo, medio y gerencial dan a la evaluación, dejando en un promedio de 4.45 su calificación sobre un total de 5.0. Se destaca la importancia que tiene en la empresa grande, en especial para el nivel de los trabajadores operarios.

Otra de las variables a medir se relaciona con la capacitación realizada en las organizaciones, se indagó sobre el valor que daban las empresas a la capacitación, en este sentido se preguntó sobre quiénes asumían los costos de los procesos de capacitación.

Se encontró que las empresas son las principales financiadoras de los procesos de capacitación en todos los niveles, se destaca el aporte importante que dan los proveedores en este sentido (20\% en promedio) y el pago que realizan los niveles gerenciales de su propia formación empresarial (ver Figura 8).

De igual modo se valoró la capacidad que tendría un sistema de capacitación laboral como mecanismo de motivación de los empleados como se aprecia en la Figura 9.

Se encuentra que en el nivel operativo los em0pleados sienten que la motivación es muy baja y solamente un $10 \%$ de aquellos que pertenecen a las empresas pequeñas señaló que la empresa los motivaba con capacitación. En tanto en los niveles de supervisor y gerencial para los tres tamaños empresariales, los funcionarios consideraron que una motivación importante (en promedio 15\%) se debía a la participación en cursos, talleres y otras actividades. Llama la atención en los tres niveles el porcentaje de personas que consideran que la empresa no los motiva, en un rango de 5 a $10 \%$. 
Figura 7. Evaluación como indicador de una competencia

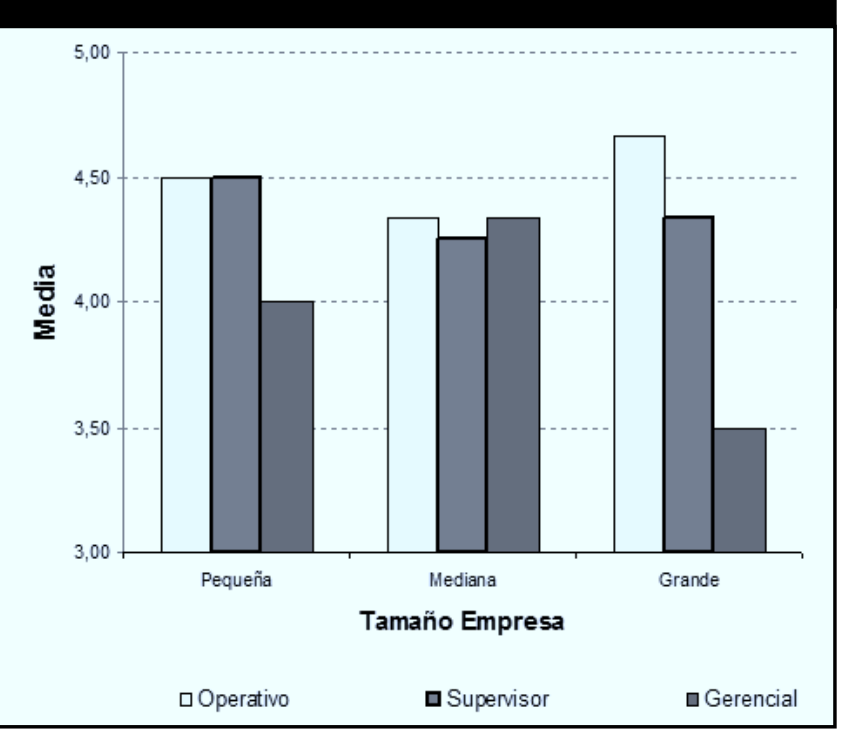

Figura 8. Financiación de la capacitación por niveles

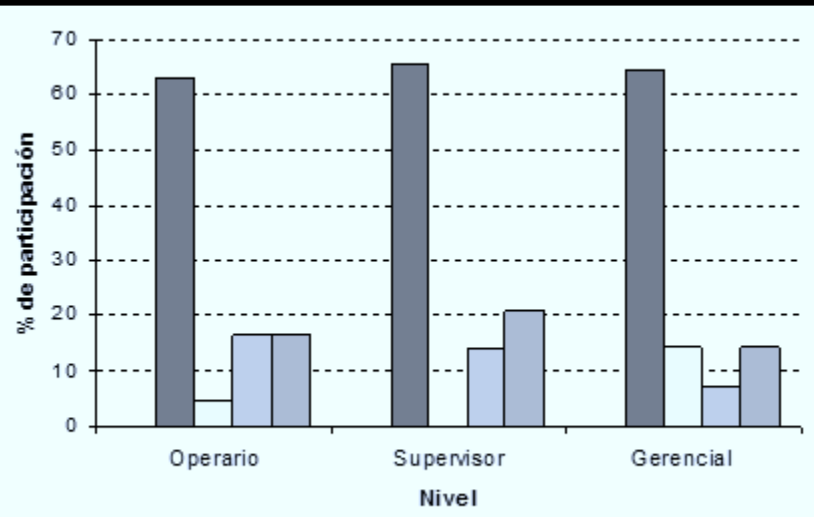

$\square$ Cofinanciación $\square$ Empresa $\square$ Empleado $\square$ Convenio $\square$ Proveedores

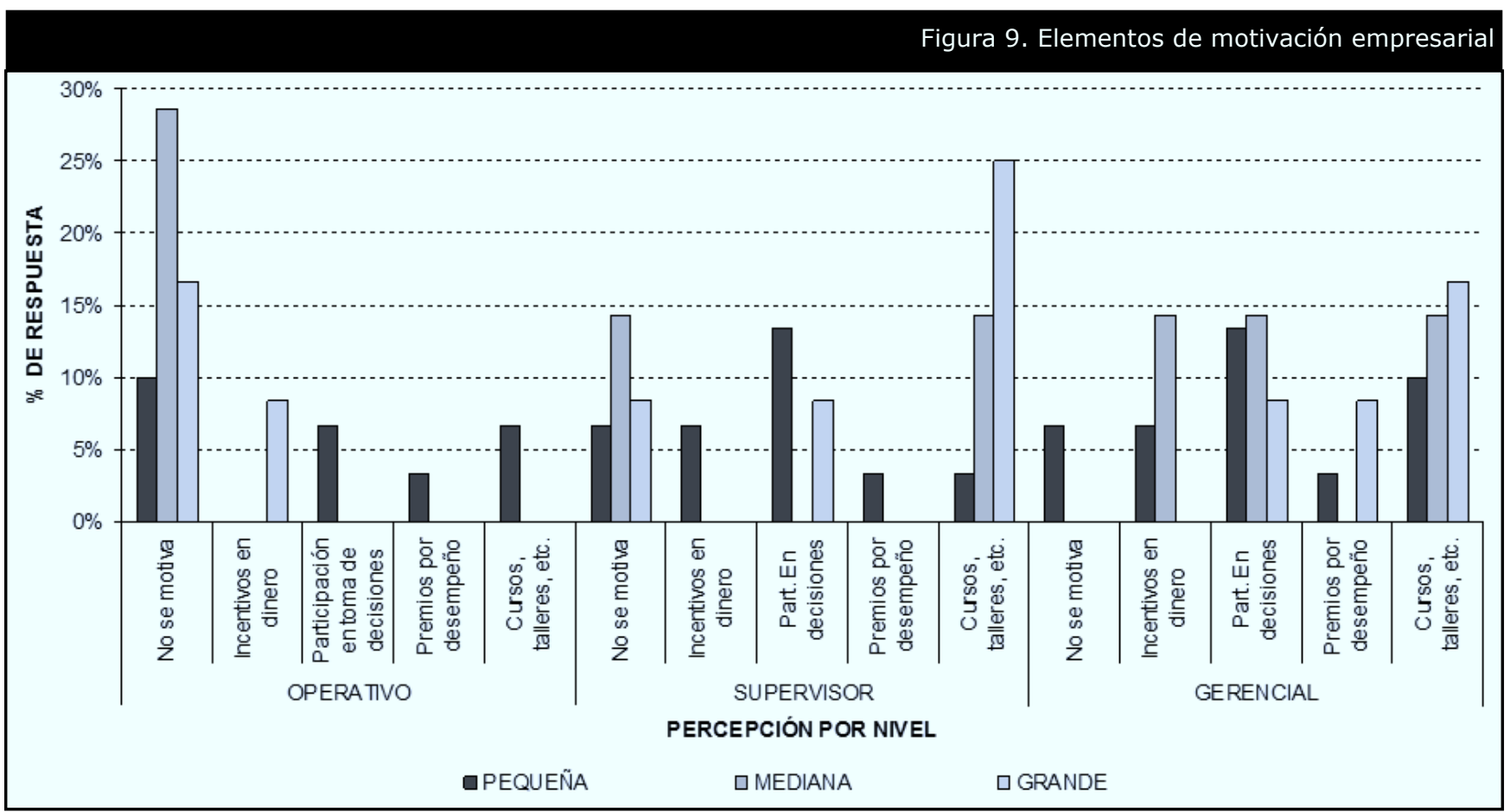


Finalmente se les preguntó sobre las ventajas y desventajas que podría traer la implementación de un sistema de competencias laborales en cada organización, los principales resultados se recogen en la Tabla 3 .

Tabla 3. Ventajas y dificultades en la implementación de un SCL

\begin{tabular}{|c|c|}
\hline Ventajas & Dificultades \\
\hline $\begin{array}{l}\text { Mejoramiento en el } \\
\text { desempeño del personal }\end{array}$ & $\begin{array}{l}\text { Dificultad en la comprensión } \\
\text { de las normas }\end{array}$ \\
\hline $\begin{array}{l}\text { Aumento de la } \\
\text { productividad y la calidad }\end{array}$ & $\begin{array}{l}\text { Existencia de demasiadas } \\
\text { calificaciones para el } \\
\text { personal }\end{array}$ \\
\hline $\begin{array}{l}\text { Mejoramiento del ingreso } \\
\text { laboral }\end{array}$ & $\begin{array}{l}\text { Incompatibilidad entre } \\
\text { las normas y la realidad } \\
\text { productiva de la empresa }\end{array}$ \\
\hline $\begin{array}{l}\text { Reconocimiento social } \\
\text { de las habilidades del } \\
\text { personal }\end{array}$ & $\begin{array}{l}\text { Demasiados trámites para el } \\
\text { proceso de certificación }\end{array}$ \\
\hline $\begin{array}{l}\text { Satisfacción y motivación } \\
\text { con el trabajo realizado }\end{array}$ & $\begin{array}{l}\text { Resistencia del personal a la } \\
\text { calificación: competente-aún } \\
\text { no competente }\end{array}$ \\
\hline $\begin{array}{l}\text { Acercamiento del sector } \\
\text { educativo con la empresa }\end{array}$ & $\begin{array}{l}\text { En caso de ser calificado } \\
\text { como aún no competente, } \\
\text { el personal puede } \\
\text { desmotivarse frente al } \\
\text { trabajo }\end{array}$ \\
\hline $\begin{array}{l}\text { Claridad en la definición } \\
\text { de los objetivos de la } \\
\text { capacitación }\end{array}$ & $\begin{array}{l}\text { Dificultad para validar las } \\
\text { competencias obtenidas } \\
\text { por estudios anteriores del } \\
\text { personal }\end{array}$ \\
\hline $\begin{array}{l}\text { Mejoramiento de la } \\
\text { relación costo- beneficio } \\
\text { en la capacitación }\end{array}$ & $\begin{array}{l}\text { Inestabilidad en las } \\
\text { relaciones internas de la } \\
\text { empresa }\end{array}$ \\
\hline $\begin{array}{l}\text { Mejoramiento en el } \\
\text { desempeño del personal }\end{array}$ & $\begin{array}{l}\text { Dificultad en la comprensión } \\
\text { de las normas }\end{array}$ \\
\hline
\end{tabular}

2.2 Posibles aportes del estudio para la adecuada implementación de las competencias laborales en el sector

A partir del trabajo realizado se concluye que las empresas de la muestra pertenecientes a la industria de la comunicación gráfica, cuentan con una población de trabajadores, en su mayoría operarios permanentes y una proporción considerable de administrativos, siendo el número de supervisores menor a los dos anteriores. Esta información lleva a considerar que al iniciar un proceso de implementación de un sistema de competencias laborales, la demanda de capacitación será permanente en este campo. En cuanto a la población de trabajadores temporales, son empleados para procesos que no requieren mayor especialización, como la encuadernación y los acabados, constituyen un mercado potencial en el que se pueden buscar mecanismos para implementar el sistema, con el fin de brindar a estos trabajadores el proceso de certificación.

De las variables analizadas en el estudio, se destaca el entorno formal en el que las relaciones con los diferentes agentes tanto internos como externos a las organizaciones, se constituyen en factor importante en la implementación de un sistema de competencias laborales En cuanto a dichos aspectos se concluye que en las empresas participantes, la información del manejo de las relaciones con diferentes agentes externos como son, proveedores, clientes y distribuidores, no es conocida por las personas de las empresas; por su parte, en el análisis y anticipación de las necesidades de los agentes internos a ellas, los empleados, las empresas medianas y grandes mantienen una percepción más optimista que las empresas pequeñas. Esto permite proponer el reforzamiento de los mecanismos de comunicación formal que faciliten el flujo de la información a todas las instancias de las empresas, así como mantener el interés que tienen por las necesidades de los trabajadores, a fin de establecer con facilidad las necesidades puntuales en la implementación de un sistema de competencias laborales.

Otro aspecto interno de las empresas al que se prestó atención en el análisis es el flujo de información en lo relacionado con la gestión humana, encontrando que en todas las empresas de la muestra, las personas consideran que la información de la gestión del factor 
humano tiende a ser conocida por los miembros de las mismas, es decir, hay claridad en las políticas que atañen al personal, tanto en los diferentes niveles como en los tamaños de empresa. De la misma forma, como parte de dicha gestión, las decisiones que se toman en cuanto a la capacitación y formación de las personas son percibidas como el producto de la concertación de los miembros, aunque también se percibe que la participación del personal no es total.

La evaluación de las competencias laborales en cada función productiva fue una de las variables fundamentales en la investigación, este aspecto se analizó con especial cuidado y se encontró que en la muestra de empresas, existe una tendencia en el nivel operativo a asignar calificaciones más favorables a dichas competencias, en contraste con los otros dos niveles -supervisor y gerencial-, que asignaron puntajes mucho más bajos, en especial, el gerencial. Esta diferencia de percepciones se debe al conocimiento diferente de la técnica en cada nivel y la diferencia de criterios técnicos en el momento de evaluar las funciones productivas.

De otro lado, cada proceso fue evaluado en forma independiente, hecho que refleja la individualidad que tienen los procesos, a pesar de ser considerados como eslabones dependientes en una misma cadena productiva. De estos resultados se concluye que la evaluación de las competencias laborales en cada empresa y función productiva ha de hacerse siguiendo una metodología que refleje el saber de cada persona, como el criterio técnico de las personas los demás niveles, haciendo que el proceso evaluativo sea integral de participación y aprendizaje mutuo y permanente.

Se observó, además, la tendencia en la mayoría de las empresas a tener contacto con procesos de calidad, el 26,6\% no habían tenido contacto con este tipo de certificaciones. El tipo de certificación más común es el ISO 9001-2000, sin embargo, existen otros certificados manejados únicamente por las empresas grandes. Lo anterior sugiere que puede haber una cultura de la calidad y de revisión de procesos ya implementada en las empresas o en proceso de hacerlo, aspectos que pueden favorecer la implementación de un sistema de competencias laborales. A pesar de este contacto con la calidad, se observa que no hay claridad de conceptos en cuanto a lo que en realidad implica en las empresas, presentándose confusión con procesos de otra índole. Este fenómeno representa un aspecto a considerar en el diseño de un modelo de competencias laborales que sea claro y que lleve a que los procesos en él involucrados mantengan una coherencia interna, de modo que no se presenten confusiones e incoherencias en el momento de su implementación.

Los antecedentes de capacitación en las empresas permiten conocer la importancia que tiene este proceso en la gestión del factor humano, los resultados obtenidos al respecto en la presente investigación dejan ver que en la mayoría de las empresas realizaron capacitación a su personal durante el año anterior, las que no realizaron este proceso son en su totalidad empresas pequeñas. Se observó, además, que la mayoría de las personas capacitadas pertenecen al nivel operativo, y para los otros dos niveles solo las empresas medianas y grandes realizan capacitación. Las actividades en las que más se capacitó a los operarios y supervisores, correspondían al proceso de impresión, por su parte, los gerentes no se capacitaron en las áreas productivas; su énfasis para capacitarse estuvo centrado en temas de gestión.

Estos aspectos dejan ver la diversidad de aspectos que involucra la capacitación en las empresas y en los que persisten los vacíos de tipo productivo en los tres niveles que deben ser atendidos por un sistema de competencias laborales. Se propone que los objetivos que hasta el momento se persiguen son percibidos de manera diferente por todos los miembros de las empresas. De otra parte, se pudo establecer que el dinero que inviertan las empresas pequeñas en este proceso es muy limitado, contrario a lo que invierten las empresas medianas y grandes, factor que puede llegar a ser limitante en la implementación del sistema.

En cuanto a la calidad de esta capacitación, se percibe como "no" adecuada, especialmente en las empresas pequeñas y medianas, presentándose como deficiente en calidad y frecuencia, lo que finalmente resulta en un proceso que no responde a las necesidades del personal.

De lo anterior se concluye que la capacitación como eslabón principal en la implementación de un sistema de competencias laborales debe tener sus objetivos alineados en todos los niveles, a fin de encontrar unidad 
y coherencia en los procesos, hecho que efectivamente sea percibido de esta manera por las personas, así como también debe asegurar la viabilidad económica del mismo a fin de que sea asequible a la mayor cantidad de empresas posible.

Esta investigación permitió observar que los trabajadores de las empresas han tenido una trayectoria prolongada en las mismas, hecho que se debe a las particularidades de los procesos productivos, en especial los de impresión, los puestos requieren de las personas un grado de especialización para desempeñarse suficientemente en cada tarea. En consecuencia, la rotación tanto interna como externa es muy baja, los trabajadores tienden a permanecer en sus puestos por mucho tiempo, perfeccionando la técnica y desarrollado las destrezas particulares que cada tarea necesita, lo que permite establecer que los procesos de capacitación pueden ser progresivos y hacerse un seguimiento de los mismos, siendo una gran ventaja para la implementación de un modelo de competencias laborales, ya que se puede dar continuidad a los procesos que se emprendan con el personal de las empresas y ver el efectivo avance de los mismos. Esta característica permite la implementación de un programa efectivo de desarrollo de competencias laborales.

Entre los aspectos contemplados en la investigación, se prestó especial atención a la evaluación del desempeño en la muestra de empresas, encontrando que es un proceso que concurre con la cultura organizacional y refleja efectivamente la competencia laboral del personal.

Por otra parte, esta evaluación está siendo percibida de manera distinta en los diferentes niveles, hecho que manifiesta el desconocimiento de los fines de dicho proceso y la necesidad de profundizar en el verdadero sentido que tiene y su beneficio para todas las personas. Este fenómeno se constituye como factor negativo, ya que las diferencias marcadas en las percepciones de la evaluación pueden obstaculizar el proceso de implementación de un sistema de competencias laborales, por lo que es un aspecto a mantener como eje fundamental, debe tener un objetivo claro para todas las personas y mantenerse como un proceso limpio y transparente, a fin de alinear las diferentes percepciones que de él pueden generarse y convertirlo en un elemento a favor del proceso de cambio.
La cultura organizacional, la cual se definió como variable crucial en la implementación de un sistema de competencias laborales, ya que de sus elementos, muchos de ellos intangibles, depende que los procesos a desarrollar se vean efectivamente adoptados por las empresas como parte de su cotidianidad y tengan un impacto significativo en la dinámica social de las empresas. Al respecto, se encontró en las empresas que a pesar de tener políticas de motivación definidas, no siempre se expresan en la percepción de los miembros de los diferentes niveles, quienes mantienen diferencias al respecto. Entre los mecanismos que utilizan las empresas para motivar a las personas se encuentran: la participación en la toma de decisiones y los cursos y talleres que se les brinda para actualizarse.

De otra parte, se encontró que las relaciones entre los diferentes niveles se mantienen en algunos casos distantes, especialmente en empresas con estructuras rígidas y verticales; aspectos como la comunicación, la independencia en la realización del trabajo, la confianza y la participación, son factores a mejorar en la mayoría de las organizaciones, especialmente en las de tamaño mediano. A pesar de lo anterior, se destaca en las empresas una cultura de aprecio hacia el trabajo y sentido de pertenencia de las personas con sus empresas, hecho que refleja una cultura favorable para los procesos de cambio, en particular los relacionados con las competencias laborales y permite proponer que este elemento debe ser incluido tanto en el análisis individual de las empresas como en el de sector, a fin de obtener un producto cada vez más ajustado a los requerimientos individuales de cada firma y que también responda a las necesidades de la industria en general.

Del estudio se destaca que la gran mayoría de las empresas participantes no ha iniciado un análisis de puestos de trabajo con el fin de implementar un sistema de competencias laborales, proceso que en general es percibido por las distintas personas como importante para ser adoptado, ya que tendrá un impacto significativo en la productividad de los trabajadores y en la competitividad de las empresas. Sin embargo, un gran número de organizaciones, en especial pequeñas, no estarían en disposición de invertir en el mismo si esto implica altos costos en tiempo y dinero. El número de personas que las empresas estarían en capacidad de 
vincular al proceso representa un gran potencial que se encuentra concentrado en la mayoría de ellas, en especial las grandes y que tiene en los operarios su mayor volumen, sin dejar de lado a los supervisores y en especial a los gerentes, que en número superan a los anteriores. En general, las empresas estarían dispuestas, a adaptar su tiempo y personal a los procesos de implementación de un sistema de competencias laborales.

\section{Conclusiones}

A partir de la iniciativa del Centro de Desarrollo Tecnológico para la Competitividad de la Industria de la Comunicación Gráfica (Cigraf), en desarrollo del proyecto "Generación de un sistema de información para Cigraf, análisis de mercado y desarrollo de programas curriculares basados en competencias laborales para la industria de la comunicación gráfica”, se desarrolló la presente investigación, que se ha constituido como un estudio de carácter exploratorio debido a la inexistencia de investigaciones similares de este tipo en la industria gráfica. Debido a esta particularidad, las conclusiones que se presentan corresponden a una muestra de empresas que sirve como referente para el diseño de un modelo basado en competencias laborales que se adapte a las necesidades del sector gráfico colombiano.

Como se pudo observar, se denota una gran oportunidad para la creación de un programa de competencias laborales, en donde se percibe como una posibilidad para desarrollar el sector, mejorar la calidad, integrar procesos, disminuir las demoras y los desechos y motivar el talento humano dándole sostenibilidad al sector.

Otra conclusión importante deriva del hecho de reconocer que no existe una empresa igual a otra, por ello se requieren planes hechos a la medida para cada una de ellas. Es necesario entonces generar procesos de formación y evaluación independientes, para cada trabajador y empresa, ajustándola a sus propias necesidades.

\section{Bibliografía}

1. Amaya, Carmen; Montoya, Alexandra; Castellanos, Oscar. (2005). Estudio exploratorio de la demanda en competencias laborales para la industria gráfica. Bogotá: Universidad Nacional de Colombia.
2. Barrios, Oscar. (2009). Taller de planificación y diseño de una asignatura técnico profesional.

3. Candiano, Roberto. (2005). Instituto la Formación Profesional Gráfica: una herramienta esencial para el desarrollo de la industria de la comunicación gráfica. Insitituto de Artes Gráficas de Oaxaca-México, Fundación Gutenberg. Buenos Aires (Argentina). [Disponible en:] http://www.conlatingraf.org/inside/ cifag/GUTEN/presentacion.pdf

4. Cardona, Pablo. (2003). Evaluating And Developing Management Competencies, lese, Professor Universidad de Navarra Barcelona, febrero. [Disponible en:] Http://Www.Strategylinksolutions. Com/Peoplelink/Notilink/Articulos/Competencia. pdf.

5. Cariola, Leonor. (1997). Competencias generales, competencias laborales y currículum. En: Competitividad, redes productivas y competencias laborales. Montevideo: OIT.

6. Catalano, Ana, et ál. (2010) Diseño curricular basado en competencias. [Disponible en:] http://www. cinterfor.org.uy/public/spanish/region/ampro/ cinterfor/publ/dis_curr/pdf/cap4.pdf

7. Cinterfor/OIT (2001). El enfoque de competencia laboral. Montevideo.

8. Cinterfor/OIT. (2010). Competencias laborales. [Disponible en:] http://www.cinterfor.org.uy/public/ spanish/region/ampro/cinterfor/temas/complab/ index.htm

9. Conocer. (1997). Elaboración del mapa funcional; guía técnica, mimeo. México: Conocer.

10. Conocer. Ibarra, Agustín. (1998) Seminario Andino sobre Formación Basada en Competencia Laboral. Bogotá.

11. Dharavath, Naik. (2004). Identification of the Skills Needed by Workers in Various Segments of the Graphic Communications Industry. Journal of Industrial Technology, 20 (3), June 2004 through August 2004 [Disponible en:] www.nait.org

12. Hernández Sampieri, Roberto et ál. (1991). Metodología de la investigación editorial. Mcgraw Hill: $501 \mathrm{p}$. 
13. Mertens, Leonard, (2000) Productividad en las organizaciones. Montevideo, Cinterfor/OIT.

14. Mertens, Leonard. (1996) Competencia laboral: sistemas, surgimiento y modelos. Montevideo: Cinterfor, $119 \mathrm{p}$.

15. Ministerio de Educación de Chile. (2003). Manual para la elaboración de módulos de formación técnica con enfoque de competencias laborales. Santiago. Disponible en: www.mineduc.cl.

16. Miranda, Martin. (2006). Taller diseño curricular y formación por competencias laborales. Bogotá.

17. Norton, RE., \& Moser, J. (2008). Dacum Handbook (3rd Ed.). Columbus, Oh: Center On Education And Training For Employment. The Ohio State University.

18. Novick, Martha. (1997). Una mirada integradora de las relaciones entre empresas y competencias laborales en América Latina. En: Competitividad, redes productivas y competencias laborales. Montevideo: OIT.

19. RedGráfica Latinoamérica. (2007). Normas de competencias laborales para la industria gráfica en Colombia. [Disponible en:] http://redgrafica.com/ Normas-de-Competencias-Laborales.

20. Romano, Frank; Fawcett, Roger; Soom, Marnie. (2003). An investigation into printing industry Demographics. Printing Industry Center, Sloan Foundation Center.

21. Suárez Martínez, Roberto Andrés; Castellano Domínguez, Óscar Fernando. (2006). Bases conceptuales e impacto de la implementación de las competencias laborales en la relación individuoorganización. Modelo de aplicación en la industria gráfica colombiana. Cuadernos Administración. Bogotá (Colombia), 19 (31): 81-101, enero-junio.

22. The Canadian Printing Industries Sector Council (CPISC). (2008). The Skills and Technology Roadmap for the Printing and Graphic Communications Industry. Canada [Disponible en:] www.cpisc-csic.ca 